\title{
关于一维随机环境中非最近邻居的随机游动 的尾估计
}

高志强

北京师范大学数学科学学院, 数学与复杂系统教育部重点实验室, 北京 100875

E-mail: gaozq@bnu.edu.cn

收稿日期: 2008-11-19; 接受日期: 2009-01-07

教育部留学回国人员科研启动基金 (批准号: [2008]890) 资助项目

摘要本文研究了一类一维随机环境中非最近邻居的随机游动, 在暂留的情况下, 给出 了它的速度, 并进一步研究了其偏离速度的尾概率的估计, 证明了这个尾概率是以多项式 的速率衰减, 给出了这个指数. 我们的结果是 Zeitouni 及其合作者在 1996 年的文章中结果 的推广. 在证明中我们用到了随机矩阵乘积的大偏差估计及随机环境中多型分支过程的总 人口数的尾概率估计和矩量估计.

关键词随机环境中的随机游动 大偏差尾估计

$\operatorname{MSC}(2000)$ 主题分类 $60 \mathrm{~J} 15,60 \mathrm{~F} 10,60 \mathrm{~J} 80$

\section{1 引言}

本文将考虑在 $\mathbb{Z}$ 上的随机环境中非最近邻居的随机游动的大偏差.

我们先来给出这个模型的明确描述. 设 $g$ 是一个整数, $\left\{\xi_{x}\right\}_{x \in \mathbb{Z}}$ 是一列取值于 $H$ 的独立 同分布 (简记为 i.i.d.) 的随机向量 (对于任意 $x, \xi_{x}=\left(\beta_{x}^{g}, \ldots, \beta_{x}^{1}, \alpha_{x}\right)$ ), 其中 $H$ 定义如下:

$$
H=\left\{\left(p_{-g}, \ldots, p_{-1}, p_{1}\right): p_{-g}+\cdots+p_{1}=1, p_{-g} p_{1}>0,0<p_{i}<1\right\} \subset(0,1)^{g+1} .
$$

对于任意 $\xi \in H$, 记 $X=\left\{X_{n}\right\}_{n \geqslant 0}$ 是定义在 $\mathbb{Z}$ 上的 Markov 链, 且以 $X_{0}=0$ 为起点, 转移 概率定义如下:

$$
\mathbf{P}_{\xi}\left(X_{n+1}=y \mid X_{n}=x\right)= \begin{cases}\alpha_{x}, & y=x+1, \\ \beta_{x}^{k}, & y=x-k, \quad k=1,2, \ldots, g, \\ 0, & \text { 否则. }\end{cases}
$$

我们称 $\xi$ 满足椭圆型条件, 如果:

$$
\exists \epsilon>0, \quad \forall z \in\{1, \ldots, g\}, \quad \beta_{x}^{g} / \alpha_{x} \geqslant \epsilon .
$$


符号 $\mathbf{P}_{\xi}$ 表示在给定环境 $\xi$ 下在轨道空间上的测度. $(X, \xi)$ 是随机环境中的随机游动 (简记 为 RWRE) 的一个例子, 分布律为 $\mathbf{P}=\int \mathbf{P}_{\xi} \Xi(\mathrm{d} \xi)$.

当 $g=1$ 时, 这个模型就是已经被广泛研究的最近邻居的随机环境中的随机游动. Solomon 在文献 [1] 中证明了常返性准则和大数定律. 在暂留情况下, 文献 [2] 给出了明确 的极限定理; 在常返情况下, 这一随机游动的运动行为在文献 [3, 4] 中已有描述. 进一步, 对 于固定环境下和依环境平均下 $n^{-1} X_{n}$ 的大偏差已经研究过, 如文献 [5-10]. 关于这一类最近 邻居的随机环境中的随机游动进展的综述及更多的文献, 请参阅文献 [11-13].

关于一般的非最近邻居的随机环境中的随机游动的最新进展, 请参阅文献 [14-18].

在本文中, 我们将考虑 $g \geqslant 2$ 的情形, 即非最近邻居的随机环境中的随机游动. 我们将研 究在依环境 $\xi$ 平均下 (即在测度 $\Xi$ 下) $X_{n} / n$ 的尾估计. 为了简单, 我们取 $g=2$, 但我们的 证明可以很容易就推广到 $g>2$.

\section{2 定义及结果}

我们考虑前面由式 (1) 定义的模型. 假设 $g=2$, 且记 $\beta_{n}^{1}=\beta_{n}, \beta_{n}^{2}=\gamma_{n}$. 我们将用到下 面的随机矩阵 $\left\{A_{n}\right\}$ :

$$
A_{n}=\left(\begin{array}{cc}
\frac{\beta_{n}+\gamma_{n}}{\alpha_{n}} & \frac{\gamma_{n}}{\alpha_{n}} \\
1 & 0
\end{array}\right) .
$$

记 $T_{\mu}$ 为包含 $A_{n}$ 的所有取值的最小半群. 对于 $\lambda \geqslant 0$, 定义

$$
\phi(\lambda)=\lim _{n}\left\{\mathbb{E}\left(\left\|A_{n} \cdots A_{1}\right\|^{\lambda}\right)\right\}^{1 / n},
$$

$\lambda_{\infty}=\sup \{\lambda \geqslant 0 ; \phi(\lambda)<+\infty\}$. 那么容易看到 $\log \phi(\lambda)$ 是 $\left[0, \lambda_{\infty}\right)$ 上的凸函数, 我们稍后将用 到这一事实.

定义 1 称半群 $T \subset G L\left(\mathbb{R}^{d}\right)$ 为强不可约的, 如果不存在 $\mathbb{R}^{d}$ 的有限个真子空间的并是 不变的.

定义 2 称半群 $T \subset G L\left(\mathbb{R}^{d}\right)$ 为吸收的, 如果存在 $T$ 中的序列 $\left\{M_{n}\right\}_{n \geqslant 0}$ 使得 $\left\|M_{n}\right\|^{-1} M_{n}$ 收玫到秩为 1 的矩阵.

定理 1 对由式 (1) 定义的随机环境中的随机游动, 假设 $\xi$ 满足椭圆型条件 (2), $T_{\mu}$ 是 强不可约的和吸收的, 存在 $\theta>1$ 使得 $\phi(\theta)=1$. 那么 RWRE 的速度是 $\mathbf{P}$ a.s. 正的:

并且, 对任何不含 $v_{0}$ 的开集 $G \subset\left(0, v_{0}\right)$,

$$
\lim _{n \rightarrow \infty} \frac{X_{n}}{n}=v_{0}>0 \text {. }
$$

$$
\lim _{n \rightarrow \infty} \log \mathbf{P}\left(n^{-1} X_{n} \in G\right) / \log n=1-\theta ;
$$

对任何与 $\left[0, v_{0}\right]$ 不交的闭集 $F$,

$$
\limsup _{n \rightarrow \infty} n^{-1} \log \mathbf{P}\left(n^{-1} X_{n} \in F\right)<0 .
$$

\section{3 定理 1 的证明}

首先我们来证明速度是 $\mathbf{P}$ a.s. 正的. 事实上, 在文献 [16] 中已经出现这一结论, 但是我 们这里的证明用了不同的方法. 
关于 $\phi$ 的假设条件蕴含了随机游动是暂留的, 于是我们可以定义停时如下:

$$
\tau_{n}=\inf \left\{k: X_{k}=n\right\} .
$$

我们通过证明关于 $\tau_{n}$ 的大数定律从而得到关于 $X_{n}$ 的相应结论.

注意到 $\tau_{n}=\sum_{k=1}^{n}\left(\tau_{k}-\tau_{k-1}\right)$ 及 $\tau_{0}=0$, 所以我们关于所考虑模型的条件保证了 $\left\{\tau_{k}-\right.$ $\left.\tau_{k-1}: k=1, \ldots, n\right\}$ 是在测度 $\Xi$ 下严格稳定且遍历的 (参阅文献 [1]). 由熟知的遍历定理可 知

$$
\lim _{n \rightarrow \infty} \frac{\tau_{n}}{n}=\mathbb{E} \tau_{1} .
$$

为了计算量 $\mathbb{E} \tau_{1}$, 我们将使用类似于文献 [2] 中的技巧. 我们引入与 $\tau_{1}$ 相关的随机环境 中的分支过程. 为此, 先给出下列记号:

$$
\begin{aligned}
& N^{+}=\#\left\{\text { 在 }\left[0, \tau_{1}\right) \text { 内向右移动的次数 }\right\}, \\
& N^{-}=\#\left\{\text { 在 }\left[0, \tau_{1}\right) \text { 内向右步长为 } 1 \text { 的移动的次数 }\right\}, \\
& N^{2-}=\#\left\{\text { 在 }\left[0, \tau_{1}\right) \text { 内向右步长为 } 2 \text { 的移动的次数 }\right\},
\end{aligned}
$$

其中 \# 表示所示集合的基数. 我们有

$$
1=X_{\tau_{1}}=N^{+}-2 N^{2-}-N^{-}, \quad \tau_{1}=N^{+}+N^{2-}+N^{-},
$$

于是

$$
\tau_{1}=1+3 N^{2-}+2 N^{-} .
$$

对任意 $x \in \mathbb{Z}^{-}$, 记

$$
\begin{aligned}
& U_{1, x}=\#\left\{k \in\left\{0,1, \ldots, \tau_{1}-1\right\}: X_{k}=x, X_{k+1}=x-1\right\}, \\
& U_{2, x}=\#\left\{k \in\left\{0,1, \ldots, \tau_{1}-1\right\}: X_{k}=x, X_{k+1}=x-2\right\}, \\
& V_{x}=U_{1, x}+U_{2, x},
\end{aligned}
$$

则我们有

$$
N^{2-}=\sum_{x=-\infty}^{0} U_{2, x}, \quad N^{-}=\sum_{x=-\infty}^{0} U_{1, x}, \quad \tau_{1}=1+\sum_{x=-\infty}^{0}\left(2 V_{x}+U_{2, x}\right) .
$$

注意每次从 $x$ 到 $x-1$ 或到 $x-2$ 的移动必定发生在两次从 $x$ 到 $x+1$ 的成功移动之间. 当 对于某个 $t_{0}$ 使得 $X_{t_{0}}=x$, 那么在给定条件 $\xi$ 和 $X_{0}, X_{1}, \ldots, X_{t_{0}}$ 下, 在下一次从 $x$ 移动到 $x+1$ 之前, 从 $x$ 到 $x-1$ 移动 $m-r$ 次且从 $x$ 到 $x-2$ 移动 $r$ 次的条件概率是 $\alpha_{x} C_{m}^{r} \beta_{x}^{m-r} \gamma_{x}^{r}$. 所以给定环境 $\xi$ 下, 序列

$$
\begin{aligned}
W_{0} & =(1,0,0), \\
W_{1} & =\left(V_{0}, U_{2,0}, U_{2,1}\right), \quad\left(\text { 这里定义 } U_{2,1}=0\right) \\
& \vdots \\
W_{k} & =\left(V_{-k+1}, U_{2,-k+1}, U_{2,-k+2}\right),
\end{aligned}
$$


组成了非齐次多态型的分支过程, 其分布如下:

$$
\begin{aligned}
& \mathbf{P}\left(W_{k}=(m, r, 0) \mid W_{k-1}=(1,0,0), \xi\right)=\alpha_{-k+1} C_{m}^{r} \beta_{-k+1}^{m-r} \gamma_{-k+1}^{r}, \\
& \mathbf{P}\left(W_{k}=(m, r, 0) \mid W_{k-1}=(0,0,1), \xi\right)=\alpha_{-k+1} C_{m}^{r} \beta_{-k+1}^{m-r} \gamma_{-k+1}^{r}, \\
& \mathbf{P}\left(W_{k}=(0,0,1) \mid W_{k-1}=(1,1,0), \xi\right)=1 .
\end{aligned}
$$

当 $\xi$ 也是随机变化时, $\left\{W_{n}\right\}$ 就成为随机环境中的分支过程. 因为 $\left\{\xi_{n}\right\}$ 是独立同分布的, $\left\{W_{n}\right\}$ 与以 $Z_{0}=(1,0,0)$ 为初值, 分布律如下定义的随机环境中的分支过程 $\left\{Z_{n}\right\}$ 是同分布:

$$
\begin{aligned}
& \mathbf{P}\left(Z_{k+1}=(m, r, 0) \mid Z_{k}=(1,0,0), \xi\right)=\alpha_{k} C_{m}^{r} \beta_{k}^{m-r} \gamma_{k}^{r}, \\
& \mathbf{P}\left(Z_{k+1}=(m, r, 0) \mid Z_{k}=(0,0,1), \xi\right)=\alpha_{k} C_{m}^{r} \beta_{k}^{m-r} \gamma_{k}^{r}, \\
& \mathbf{P}\left(Z_{k+1}=(0,0,1) \mid Z_{k}=(1,1,0), \xi\right)=1 .
\end{aligned}
$$

我们记 $M_{n}$ 为再生律的期望矩阵. 它们是 $d=g+1=3$ 阶的矩阵, 定义 $M_{n}(i, j)$ 为第 $j$ 型 粒子产生第 $i$ 型粒子的个数的期望. $\left\{M_{n}\right\}_{n \geqslant 1}$ 是一列独立同分布的随机矩阵, 从式 (11) 可 算得 $M_{n}$ 如下:

$$
M_{n}=\left(\begin{array}{ccc}
\frac{\beta_{n}+\gamma_{n}}{\alpha_{n}} & 0 & \frac{\beta_{n}+\gamma_{n}}{\alpha_{n}} \\
\frac{\gamma_{n}}{\alpha_{n}} & 0 & \frac{\gamma_{n}}{\alpha_{n}} \\
0 & 1 & 0
\end{array}\right) .
$$

那么我们就得到

$$
\mathbb{E}_{\xi}\left(Z_{k}\right)=\mathbb{E}_{\xi}\left(Z_{k} \mid Z_{k-1}\right) \mathbb{E}_{\xi}\left(Z_{k-1}\right)=M_{k-1} \mathbb{E}_{\xi}\left(Z_{k-1}\right)=M_{k-1} \cdots M_{0} Z_{0} .
$$

综合前面讨论, 我们有

$$
\mathbb{E}\left(\tau_{1}\right)=1+\left\langle\ell, \mathbb{E} \sum_{n=1}^{\infty} \mathbb{E}_{\xi}\left(Z_{n}\right)\right\rangle=1+\left\langle\ell, \sum_{n=1}^{\infty} \mathbb{E}\left(M_{n-1} \cdots M_{0}\right) Z_{0}\right\rangle,
$$

其中 $\ell=(2,1,0)$, 上式中级数的收玫性是由条件 $\phi(1)<\phi(\theta)=1$ 及下面的关系保证的. 下面 来看一下 $A_{n}$ 与 $M_{n}$ 的关系: 假设 $T$ 是从 $\mathbb{R}^{3}$ 到 $\mathbb{R}^{2}$ 的线性映射,

$$
T=\left(\begin{array}{lll}
1 & 0 & 1 \\
0 & 1 & 0
\end{array}\right),
$$

可以验证 $T M_{n}=A_{n}^{t} T$, 进一步,

$$
T M_{n} M_{n-1} \cdots M_{0}=A_{n}^{t} A_{n-1}^{t} \cdots A_{0}^{t} T .
$$

注意到对 $u \geqslant 0,\|T u\|_{1}=\|u\|_{1}$, 其中 $\|u\|_{1}=\sum_{i=1}^{d} u_{i}$. 而 $\|\cdot\|$ 与 $\|\cdot\|_{1}$ 的等价性保证了 $\left\|M_{n} M_{n-1} \cdots M_{0}\right\|$ 等价于 $\left\|A_{n}^{t} A_{n-1}^{t} \cdots A_{0}^{t}\right\|$. 所以

$$
\phi(\lambda)=\lim _{n}\left\{\mathbb{E}\left(\left\|M_{n} \cdots M_{1}\right\|^{\lambda}\right)\right\}^{1 / n} .
$$

为了得到 $X_{n}$ 的大数定律, 引入与 $n$ 相关的量 $r(n)$, 使得

$$
\tau_{r(n)} \leqslant n<\tau_{r(n)+1} .
$$

容易看到 $X_{n}<r(n)+1$ 及 $r(n) \leqslant X_{n}+2\left(n-\tau_{r(n)}\right)$. 所以可推出:

$$
\frac{r(n)}{n}-2\left(1-\frac{\tau_{r(n)}}{n}\right)<\frac{X_{n}}{n}<\frac{r(n)+1}{n} .
$$


由于 $X_{n} \rightarrow+\infty \mathbf{P}$ a.s., $r(n) \rightarrow \infty \mathbf{P}$ a.s. 及 $\frac{\tau_{r(n)}}{n} \rightarrow 1$ a.s., 那么可得

$$
\lim _{n \rightarrow+\infty} \frac{r(n)}{n}=\lim _{n \rightarrow+\infty} \frac{X_{n}}{n} .
$$

又由于 $\frac{r(n)}{n}=\frac{\tau_{r(n)}}{n} \cdot \frac{r(n)}{\tau_{r(n)}}$, 故而 $\lim _{n} \frac{r(n)}{n}=\lim _{n} \frac{n}{\tau_{n}}$. 所以得到

下面来证明式 (6).

$$
\lim _{n \rightarrow+\infty} \frac{X_{n}}{n}=\lim _{n \rightarrow+\infty} \frac{n}{\tau_{n}}=\frac{1}{\mathbf{E}\left(\tau_{1}\right)} .
$$

令 $\bar{X}_{n}$ 表示初值为 0 , 与 $\xi$ 相关的转移概率定义与 (1) 中相同, 但定义 $\xi_{0}=\xi_{1}=(1,0,0)$. 令

$$
\bar{\tau}_{n}=\inf \left\{k: \bar{X}_{k}=n\right\} .
$$

下面关于 $\bar{\tau}_{n}$ 的尾估计将会用在 $\mathbf{P}\left(n^{-1} X_{n} \in G\right)$ 的下界估计中.

引理 1

$$
\mathbf{P}_{\xi}\left(\bar{\tau}_{k} \geqslant n\right) \geqslant\left(1-\left(\mathbf{e}_{1}^{t} A_{k-1,1} \mathbf{e}_{1}\right)^{-1}\right)^{n} .
$$

为证明这个引理, 我们需要下面的结果. 考虑整数 $(a, b, k)$, 并定义

$$
\mathbf{P}_{k}\{a, b,-\}:=\mathbf{P}\left\{X_{n} \text { 在到达 }[b,+\infty) \text { 之前先到达 }(-\infty, a] \mid X_{0}=k, \xi\right\} \text {, }
$$

那么从文献 [16] 中得到

\section{引理 2}

$$
\mathbf{P}_{k}\{a, b,-\}=\left(\sum_{l=k}^{b-1} \mathbf{e}_{1}^{t} A_{l, a+1} \mathbf{e}_{1}\right)\left(1+\sum_{l=a+1}^{b-1} \mathbf{e}_{1}^{t} A_{l, a+1} \mathbf{e}_{1}\right)^{-1},
$$

其中 $A_{l, a+1}=A_{l} A_{l-1} \cdots A_{a+1}$.

\section{引理 1 的证明 由引理 2 ,}

$$
\begin{aligned}
\mathbf{P}_{\xi} & \left(\bar{X}_{n} \text { 在到达 } k \text { 之前先到达 }(-\infty, 0]: \bar{X}_{0}=0\right) \\
& =\mathbf{P}_{1}\{0, k,-\} \\
& =\left(\sum_{l=1}^{k-1} \mathbf{e}_{1}^{t} A_{l, 1} \mathbf{e}_{1}\right)\left(1+\sum_{l=1}^{k-1} \mathbf{e}_{1}^{t} A_{l, 1} \mathbf{e}_{1}\right)^{-1} \\
& =1-\left(1+\sum_{l=1}^{k-1} \mathbf{e}_{1}^{t} A_{l, 1} \mathbf{e}_{1}\right)^{-1} \\
& \geqslant 1-\left(\mathbf{e}_{1}^{t} A_{k-1,1} \mathbf{e}_{1}\right)^{-1}
\end{aligned}
$$

那么由 $\mathbf{P}_{\xi}\left(\bar{\tau}_{k} \geqslant n\right) \geqslant\left(\mathbf{P}_{1}\{0, k,-\}\right)^{n}$ 即可得到式 (17).

对 $y \in \mathbb{Z}$, 令 $\tau_{y}=\min \left\{n: X_{n}=y\right\}$. 那么下个引理给出了 RWRE 从 $y$ 出发向左运动的最 长距离 $L_{y}=\max \left\{y-X_{n}: n \geqslant \tau_{y}\right\}$ 的一个指数型尾估计. 这个估计在后面对 $\mathbf{P}\left(n^{-1} X_{n} \in G\right)$ 上下界的证明中有关键作用.

引理 3 对于 $0<\varepsilon<1-\phi(1)$, 存在 $k_{0} \geqslant 1$ 使得对于任意 $y \in \mathbb{Z}$ 与 $k \geqslant k_{0}$,

$$
\mathbf{P}\left(L_{y} \geqslant k\right) \leqslant(1-\phi(1)-\varepsilon)^{-1}(\phi(1)+\varepsilon)^{k} .
$$

证明 注意对任意 $k \geqslant 1$,

$$
\Theta_{y, k}=\mathbf{P}_{\xi}\left(L_{y} \geqslant k\right)=\mathbf{P}_{\xi} \text { (存在 } n>0 \text {, 使得 } X_{n} \leqslant y-k \mid X_{0}=y \text { ) }
$$


是以 $y$ 为指标的稳定过程. 由引理 2 , 可得

$$
\Theta_{0, k}=\left(\sum_{l=0}^{+\infty} \mathbf{e}_{1}^{t} A_{l,-k} \mathbf{e}_{1}\right)\left(1+\sum_{l=0}^{+\infty} \mathbf{e}_{1}^{t} A_{l,-k} \mathbf{e}_{1}\right)^{-1} .
$$

根据 $\phi(\lambda)$ 的定义, 可知存在 $k_{0}$ 使得

$$
\mathbf{E}\left\|A_{0,-k}\right\|<(\phi(1)+\varepsilon)^{k}, \quad \forall k \geqslant k_{0} .
$$

需证结论立刻由下面的事实所推出:

$$
\begin{aligned}
\mathbf{P}\left(L_{y} \geqslant k\right) & =\mathbf{E}\left(\Theta_{y, k}\right)=\mathbf{E}\left(\Theta_{0, k}\right) \leqslant \sum_{l=0}^{+\infty} \mathbf{E}\left\|A_{l,-k}\right\| \\
& \leqslant \sum_{l=0}^{\infty}(\phi(1)+\varepsilon)^{k+l}=(1-\phi(1)-\varepsilon)^{-1}(\phi(1)+\varepsilon)^{k} .
\end{aligned}
$$

我们还需要一个关于大偏差的结果:

引理 4

$$
\lim _{n \rightarrow \infty} \frac{1}{n} \log \mathbf{P}\left(n^{-1} \log \left|\mathbf{e}_{1}^{t} A_{n} A_{n-1} \cdots A_{1} \mathbf{e}_{1}\right|>y_{\theta}\right)=-y_{\theta} \theta,
$$

其中 $y_{\theta}=\phi^{\prime}(\theta)$.

证明 我们已知

$$
\log \phi(\lambda)=\lim _{n \rightarrow \infty} \frac{1}{n} \log \mathbf{E}\left(\left\|A_{1} A_{2} \cdots A_{n}\right\|^{\lambda}\right) .
$$

由于 $\mathbf{e}_{2}^{t} A_{n} A_{n-1} \cdots A_{1} \mathbf{e}_{1}=\mathbf{e}_{1}^{t} A_{n-1} A_{n-2} \cdots A_{1} \mathbf{e}_{1}$, 且 $\left\{\mathbf{e}_{1}, \mathbf{e}_{2}\right\}$ 是 $\mathbb{R}^{2}$ 的标准基, 因此

$$
\begin{aligned}
\lim _{n \rightarrow \infty} & \frac{1}{n} \log \mathbf{P}\left(n^{-1} \log \left|\mathbf{e}_{1}^{t} A_{n} A_{n-1} \cdots A_{1} \mathbf{e}_{1}\right|>y_{\theta}\right) \\
\quad= & \lim _{n \rightarrow \infty} \frac{1}{n} \log \mathbf{P}\left(n^{-1} \log \left\|A_{n} A_{n-1} \cdots A_{1} \mathbf{e}_{1}\right\|>y_{\theta}\right) .
\end{aligned}
$$

利用文献 [19] 中关于准紧算子谱分解的结论, 在文献 [20,21] 中已经证明了在我们现在的条 件下, 对任意 $x \in \mathbb{R}^{2}$,

$$
\lim _{n} \frac{1}{n} \log \mathbf{P}\left(\log \left\|A_{n} A_{n-1} \cdots A_{1} x\right\|>n y\right)=-\sup \{y t-\log \phi(t): \phi(t)<\infty\} .
$$

特别如果我们选取 $y_{\theta}=\phi^{\prime}(\theta)$, 那么

$$
\sup \{y t-\log \phi(t): \phi(t)<\infty\}=y_{\theta} \theta-\log \phi(\theta)=y_{\theta} \theta .
$$

由于 $G \subset\left(0, v_{0}\right)$ 是不含 $v_{0}$ 的开集, 所以在下面引理中只须对于 $G=(v-2 \eta, v)$ (这里 $\left.0<2 \eta<v<v_{0}\right)$ 的形式进行证明.

引理 $\mathbf{5}$ 设 $0<2 \eta<v<v_{0}$, 那么有

$$
\liminf _{n \rightarrow \infty} \log \mathbf{P}\left(n^{-1} X_{n} \in(v-2 \eta, v)\right) / \log n \geqslant 1-\theta .
$$

证明 设 $v$ 和 $\eta$ 满足引理中条件. 注意到事件 $\left\{n^{-1} X_{n} \in(v-2 \eta, v)\right\}$ 包含了事件

$$
\left\{\frac{(v-2 \eta) n}{v_{0}}<\tau_{(v-\eta) n}<n, L_{(v-\eta) n}<\eta n, \tau_{v n}>n\right\},
$$

也就是说, 随机游动大约在预期的时间到达了 $(v-\eta) n$, 且从这一点出发它向左边移动的最 大距离小于 $\eta n$, 但到时刻 $n$ 尚未到达 $v n$.

回忆由式 (5) 可推出当 $n \rightarrow \infty$ 时, $\mathbf{P}\left(\tau_{(v-\eta) n} \in\left((v-2 \eta) n / v_{0}, n\right)\right) \rightarrow 1$. 令 $\zeta=1-(v-$ $2 \eta) / v_{0}>0$. 由于 $\tau_{(v-\eta n)}$ 与事件 $\left\{\xi_{x}: x \geqslant(v-\eta) n\right\}$ 相互独立, 故而由稳定性得

$$
\mathbf{P}\left(\tau_{v n}>n \mid \tau_{(v-\eta) n} \in\left((v-2 \eta) n / v_{0}, n\right)\right) \geqslant \mathbf{P}\left(\bar{\tau}_{\eta n}>\zeta n\right) .
$$


于是根据 $\mathbf{P}\left(L_{(v-\eta) n} \geqslant \zeta n\right)$ 的指数界 (见引理 3), 我们要证式 (19) 只须证明

$$
\liminf _{n \rightarrow+\infty} \log \mathbf{P}\left(\bar{\tau}_{\eta n}>\zeta n\right) / \log n \geqslant 1-\theta .
$$

由稳定性和式 (18), 我们可得

$$
\mathbf{P}\left(\log \left|\mathbf{e}_{1}^{t} A_{k-1,1} \mathbf{e}_{1}\right| /(k-1)>y_{\theta}\right) \geqslant e^{-\left(y_{\theta} \theta+o(1)\right)(k-1)} \text {, 当 } k \rightarrow \infty .
$$

选取

$$
k=k(n)=1+\frac{\log n}{y_{\theta}},
$$

那么当 $n$ 和 $k$ 趋于 $\infty$ 时, $e^{-\left(\theta y_{\theta}+o(1)\right)(k-1)}=n^{-\theta+o(1)}$. 考虑事件

$$
\mathfrak{A}_{n}=\left\{\xi: \max _{m=0,1, \ldots,[\eta n / k-1]-1}(k-1)^{-1} \log \left|\mathbf{e}_{1}^{t} A_{(m+1) k-1, m k+1}(\xi) \mathbf{e}_{1}\right| \geqslant y_{\theta}\right\} .
$$

由于 $\left\{\log \left(\mathbf{e}_{1}^{t} A_{(m+1) k-1, m k+1} \mathbf{e}_{1}\right)\right\}_{m \geqslant 0}$ 是独立同分布的随机变量, 我们可以从式 (21) 推出

$$
\liminf _{n \rightarrow \infty} \frac{1}{\log n} \log \mathbf{P}\left(\mathfrak{A}_{n}\right) \geqslant \liminf _{n \rightarrow \infty} \frac{1}{\log n} \log \left(\frac{\eta n}{k(n)} n^{-\theta}\right)=1-\theta .
$$

根据

$$
m^{*}=\min \left\{m \geqslant 0:(k-1)^{-1} \log \left|\mathbf{e}_{1}^{t} A_{(m+1) k-1, m k+1} \mathbf{e}_{1}\right| \geqslant y_{\theta}\right\}
$$

来分解事件 $\mathfrak{A}_{n}$, 并且忽略 Markov 链 $X_{n}$ 在 $\left[m^{*} k, m^{*} k+k\right)$ 之外所用的时间, 那么我们可以 由稳定性得到

$$
\mathbf{P}\left(\bar{\tau}_{\eta n}>\zeta n \mid \mathfrak{A}_{n}\right) \geqslant \inf _{\xi}\left\{\mathbf{P}_{\xi}\left(\bar{\tau}_{k}>\zeta n\right):(k-1)^{-1} \log \left|\mathbf{e}_{1}^{t} A_{k-1,1}(\xi) \mathbf{e}_{1}\right| \geqslant y_{\theta}\right\} .
$$

由引理 1 ,

$$
\begin{gathered}
\inf _{\xi}\left\{\mathbf{P}_{\xi}\left(\bar{\tau}_{k}>\zeta n\right):(k-1)^{-1} \log \left|\mathbf{e}_{1}^{t} A_{k-1,1}(\xi) \mathbf{e}_{1}\right| \geqslant y_{\theta}\right\} \\
\geqslant \inf _{z \geqslant y_{\theta}}\left(1-e^{-(k-1) z}\right)^{\zeta n+1} \geqslant\left(1-n^{-1}\right)^{\zeta n+1} .
\end{gathered}
$$

结合式 (22) 和式 (23), 我们就可证明式 (20), 从而完成了引理的证明.

对 $\mathbf{P}\left(n^{-1} X_{n} \in G\right)$ 的上界估计将通过研究停时的矩估计得到. 为此观察到 $\tau_{k}=\sum_{i=1}^{k}\left(\tau_{i}-\right.$ $\left.\tau_{i-1}\right)$ (其中 $\tau_{0}=0$ ), 即可以写成与 $\tau_{1}$ 同分布的 (相依) 随机变量 $\left(\tau_{i}-\tau_{i-1}\right)$ 的和. 记 $C_{\theta^{\prime}}=\mathbf{E}\left(\tau_{1}^{\theta^{\prime}}\right)$, 则由 Minkowski 不等式可得, 当 $k \geqslant 1$,

$$
\mathbf{E}\left(\tau_{k}^{\theta^{\prime}}\right) \leqslant C_{\theta^{\prime}} k^{\theta^{\prime}} .
$$

且由文献 [22] 中的引理 3.3 知, 当 $\theta^{\prime}<\theta$ 时 $C_{\theta^{\prime}}<\infty$.

下面开始研究 $\mathbf{P}\left(n^{-1} X_{n} \in G\right)$ 的上界.

仿照文献 [6], 我们有下面的引理.

引理 6 对任意 $v \in\left(0, v_{0}\right)$, 当 $\theta^{\prime}<\theta$ 和 $n$ 充分大时,

$$
\mathbf{P}\left(X_{n}<v n\right) \leqslant n^{1-\theta^{\prime}} .
$$

证明 这个证明与文献 [6] 中类似, 但为了描述完整以方便读者, 我们给出具体过程.

取定 $A>-\theta / \log \phi(1)$, 并设 $k=k(n)=A \log n$. 在与 $\left\{X_{t}\right\}$ 相同的概率空间上, 定义过程 $\left\{Y_{t}\right\}$ 和停时 $\widetilde{\tau}_{i k}=\min \left\{t \geqslant 0: Y_{t}=i k\right\}$, 这两个过程之间的唯一改变就是当 $t \geqslant \widetilde{\tau}_{i k}(i=0, \ldots)$ 时, $Y_{t}$ 在位置 $(i-1) k$ 和 $(i-1) k-1$ 被反射 (令 $\alpha_{i k}=1$ 及 $\alpha_{i k-1}=1$ 即可). 记 $N=[v n / k]+1$ 及 $T_{k}^{(i)}=\widetilde{\tau}_{i k}-\widetilde{\tau}_{(i-1) k}, i=1, \ldots, N$. 注意到 $T_{k}^{(i)}$ 是同分布的, 每一个都被 $\tau_{k}$ 随机控制. 记 
$T_{k}=T_{k}^{(1)}$, 则 $\mathbf{E}\left(T_{k}\right) \leqslant \mathbf{E}\left(\tau_{k}\right)$. 取定 $\lambda \in(1 / \theta, 1)$. 由式 (24) 可得 $\mathbf{E}\left(\tau_{k}^{1 / \lambda}\right)<C k^{1 / \lambda}$, 其中 $C<\infty$ 是个常数. 进而, 由 Hölder 不等式可得

$$
\mathbf{E}\left(\tau_{k}\right) \leqslant \mathbf{E}\left(T_{k}\right)+\mathbf{P}\left(L_{0} \geqslant k\right)^{1-\lambda} \mathbf{E}\left(\tau_{k}^{1 / \lambda}\right)^{\lambda} .
$$

那么由引理 3 , 当 $n$ 充分大时,

$$
\begin{aligned}
\mathbf{P}\left(X_{n}<v n\right) & \leqslant \mathbf{P}\left(X_{n} \leqslant(N-1) k\right) \\
& \leqslant \mathbf{P}\left(\sum_{i=1}^{N} T_{k}^{(i)}>n\right)+\mathbf{P}\left(\max _{i=0, \ldots, N} L_{i k} \geqslant k\right) \\
& \leqslant \mathbf{P}\left(\sum_{i=1}^{N} T_{k}^{(i)}>n\right)+n^{-\left(\theta^{\prime}-1\right)} .
\end{aligned}
$$

因为 $v^{-1}>v_{0}^{-1}=\lim \sup _{k \rightarrow \infty} k^{-1} \mathbf{E}\left(T_{k}\right)$, 所以存在充分小的正数 $\eta>0$, 使得

$$
\begin{aligned}
\mathbf{P}\left(\sum_{i=1}^{N} T_{k}^{(i)}>n\right) & \leqslant \mathbf{P}\left(\sum_{i=1}^{N}\left(T_{k}^{(i)}-\mathbf{E}\left(T_{k}\right)\right)>2 \eta n\right) \\
& \leqslant 2 \mathbf{P}\left(\sum_{i=1}^{[N / 2]}\left(T_{k}^{(i)}-\mathbf{E}\left(T_{k}\right)\right)>\eta n\right) .
\end{aligned}
$$

现在观察到 $\left\{T_{2 k}^{(2 i)}-\mathbf{E}\left(T_{k}\right)\right\}$ 是一列独立同分布零平均的随机变量. 由于 $T_{k}$ 是被 $\tau_{k}$ 随机控 制的, 从式 (24) 可以推出对任意 $\lambda \in(0, \theta)$, 有 $\mathbf{E}\left(\left|T_{k}-\mathbf{E}\left(T_{k}\right)\right|^{\lambda}\right) \leqslant C k^{\lambda}$. 于是, 由 Markov 不 等式得, 当 $\lambda \in\left(\theta^{\prime}, \theta\right)$ 及 $n$ 充分大时,

$$
\mathbf{P}\left(T_{k}-\mathbf{E}\left(T_{k}\right)>\eta n\right) \leqslant(\eta n)^{-\lambda} \mathbf{E}\left(\left|T_{k}-\mathbf{E}\left(T_{k}\right)\right|^{\lambda}\right) \leqslant n^{-\theta^{\prime}} .
$$

于是由文献 [23],

$$
\mathbf{P}\left(\sum_{i=1}^{[N / 2]}\left(T_{k}^{(2 i)}-\mathbf{E}\left(T_{k}\right)\right)>\eta n\right) \leqslant N \mathbf{P}\left(T_{k}-\mathbf{E}\left(T_{k}\right)>\eta n\right)+0.5 n^{1-\theta^{\prime}} \leqslant n^{1-\theta^{\prime}} .
$$

现在我们来证明式 (7), 这里我们仍是采用文献 [6] 中的想法.

事件 $\left\{n^{-1} X_{n} \leqslant-\eta\right\}$ 蕴含了左行的最大距离 $L_{0}$ 至少为 $\eta n$. 由引理 3 , 对每个取定 $\eta>0$, 后一事件的概率依 $n$ 的指数级衰减.

还需证明的就是对任何 $\eta>0$,

$$
\limsup _{n \rightarrow \infty} n^{-1} \log \mathbf{P}\left(n^{-1} X_{n} \geqslant v_{0}+2 \eta\right)<0 .
$$

为此我们需要下面的引理:

引理 7

$$
C_{1}:=\mathbf{E}\left[\sum_{n=0}^{\infty} 1_{\left\{X_{n} \leqslant 0\right\}}\right]<\infty .
$$

证明 仿照 Solomon ${ }^{[20]}$, 令

$G_{i j}=\mathbf{E}\left\{\right.$ 使得 $X_{n}=j$ 的 $n$ 的个数 $\mid X_{0}=0$, 固定 $\left.\xi\right\}=\mathbf{E}_{\xi}\left[\sum_{n=0}^{\infty} 1_{\left\{X_{n}=j\right\}} \mid X_{0}=i\right]$,

$G_{j}=\mathbf{E}\left\{\right.$ 使得 $X_{n}=j$ 的 $n$ 的个数 $\left.\mid X_{0}=0\right\}=\mathbf{E} G_{0 j}$,

$f_{i j}=f_{i j}^{\xi}=\mathbf{P}\left(\right.$ 存在 $n>0, X_{n}=j \mid X_{0}=i$, 固定 $\left.\xi\right)$. 
那么

$$
\mathbf{E}\left[\sum_{n=0}^{\infty} 1_{\left\{X_{n} \leqslant 0\right\}}\right]=\mathbf{E}\left[\sum_{j=-\infty}^{0} \sum_{n=0}^{\infty} 1_{\left\{X_{n}=j\right\}}\right]=\sum_{j=-\infty}^{0} G_{j}=\sum_{j=-\infty}^{0} \mathbf{E} G_{0 j} .
$$

并且, 下面的方程成立:

$$
G_{i j}=\delta_{i j}+f_{i j} G_{j j}, \quad \text { 其中 } \delta_{i j}=1, i=j ; \delta_{i j}=0, i \neq j .
$$

由于当 $i>j$ 时, $f_{i j}=\mathbf{P}\left\{X_{n}\right.$ 在到达 $+\infty$ 之前先到达 $\left.(-\infty, j] \mid X_{0}=i, \xi\right\}$, 那么由引理 2

$$
f_{i j}=\left(\sum_{l=i}^{+\infty} \mathbf{e}_{1}^{t} A_{l, j+1} \mathbf{e}_{1}\right)\left(1+\sum_{l=j+1}^{+\infty} \mathbf{e}_{1}^{t} A_{l, j+1} \mathbf{e}_{1}\right)^{-1}, \quad i>j .
$$

因为所考虑的随机游动是向右暂留的且向右步长只能为 1 , 那么当 $i<j$ 时, $f_{i j}=1$ a.e. 从而

$$
\begin{aligned}
f_{j j} & =\alpha_{j} f_{j+1, j}+\beta_{j} f_{j-1, j}+\gamma_{j} f_{j-2, j} \\
& =\left(\beta_{j}+\gamma_{j}+\sum_{l=j+1}^{+\infty} \mathbf{e}_{1}^{t} A_{l, j+1} \mathbf{e}_{1}\right)\left(1+\sum_{l=j+1}^{+\infty} \mathbf{e}_{1}^{t} A_{l, j+1} \mathbf{e}_{1}\right)^{-1} .
\end{aligned}
$$

现在在式 (30) 中取 $i=j$, 就可以得到

$$
G_{j j}=\left(1-f_{j j}\right)^{-1}=\frac{1}{\alpha_{j}}\left(1+\sum_{l=j+1}^{+\infty} \mathbf{e}_{1}^{t} A_{l, j+1} \mathbf{e}_{1}\right) .
$$

最后再结合式 (30) 可知

$$
G_{i j}=\left\{\begin{array}{l}
\frac{1}{\alpha_{j}}\left(1+\sum_{l=j+1}^{+\infty} \mathbf{e}_{1}^{t} A_{l, j+1} \mathbf{e}_{1}\right), \quad i \leqslant j, \\
\frac{1}{\alpha_{j}}\left(\sum_{l=i}^{+\infty} \mathbf{e}_{1}^{t} A_{l, j+1} \mathbf{e}_{1}\right), \quad i>j .
\end{array}\right.
$$

依环境取期望, 用与引理 3 的证明中类似的计算讨论, 即可完成式 (28) 的证明.

由这一引理可得对任意 $k \geqslant 1$,

$$
\mathbf{E}\left(\tau_{k}-\bar{\tau}_{k}\right) \leqslant C_{1},
$$

其中 $\bar{\tau}_{k}$ 定义如式 (16) 所示. 对给定 $\eta>0$, 取 $k$ 充分大使得

由式 (31) 及 $\mathbf{E} \tau_{k}=k \mathbf{E}\left(\tau_{1}\right)=k / v_{0}$, 就可得

$$
\frac{k}{v_{0}}-C_{1}-2>\frac{k}{v_{0}+\eta} .
$$

$$
\mathbf{E}\left(\bar{\tau}_{k}\right)-2>\frac{k}{v_{0}+\eta} .
$$

现在我们来考虑一个修改的环境: 对所有的 $i$, 令 $\alpha_{i k}=\alpha_{i k-1}=1$; 对其它的整数 $x$, 转 移概率定义仍与 $\xi$ 相同. 用 $\bar{\tau}_{k}^{(i)}$ 表示在这个修改的环境下, 随机游动从 $(i-1) k-1$ 出发至 到达 $i k$ 所用的步数. 令 $\bar{\tau}_{k}^{(i), M}=\bar{\tau}_{k}^{(i)} \wedge M$, 其中 $M$ 充分大使得 $\mathbf{E}\left(\bar{\tau}_{k} \wedge M\right)>\mathbf{E}\left(\bar{\tau}_{k}\right)-1$. 随 机变量 $\left\{\bar{\tau}_{k}^{(i), M}\right\}_{i \geqslant 1}$ 是独立同分布且有有界支撑的. 对于任意充分大的 $n$, 我们能找到一个整 数 $m=m(n)$ 满足

$$
\frac{n\left(v_{0}+\eta\right)}{k}<m<\frac{n\left(v_{0}+2 \eta\right)}{k} .
$$


不等式 (32) 和 (33) 蕴含了 $n<m\left(\mathbf{E}\left(\bar{\tau}_{k}^{M}\right)-1\right)$. 因为和 $\sum_{i=1}^{m} \bar{\tau}_{k}^{(i), M}$ 是被 $\tau_{k m}$ 随机控制的, 那 么由 Cramér 定理可知存在 $c>0$ 使得

$$
\begin{aligned}
\mathbf{P}\left(n^{-1} X_{n} \geqslant v_{0}+2 \eta\right) & \leqslant \mathbf{P}\left(\tau_{k m} \leqslant n\right) \\
& \leqslant \mathbf{P}\left(\sum_{i=1}^{m} \bar{\tau}_{k}^{(i), M} \leqslant m\left(\mathbf{E}\left(\bar{\tau}_{k}^{M}\right)-1\right)\right) \leqslant e^{-c m} .
\end{aligned}
$$

则由于当 $n \rightarrow \infty$ 比值 $n / m$ 是有界的, 我们就可得到需证的式 (27).

注记 在文献 $[6,7,9,10]$ 中, 给出了关于随机环境中的最近邻居的随机游动的尾估计的 完全描述. 在本文中我们只是将文献 [6] 中的部分结果推广到了一类随机环境中的非最近邻 居的随机游动. 而文献 $[7,9,10]$ 中的结论, 对本文研究的模型的相应结果还未知.

致谢在此作者真诚感谢邓冠铁教授、刘全升教授和 Emile Le Page 教授的教导和帮助. 非常感谢审稿人对原稿提出的宝贵意见和建议.

\section{参考文献}

1 Solomon F. Random walks in random environment. Ann Probab, 3: 1-31 (1975)

2 Kesten H, Kozlov M V, Spitzer F. A limit theorem for random walk in random environment. Comput Math, 30: 145-168 (1975)

3 Kesten H. The limit distribution of Sinaï's random walk in random environment. Phys A, 138: 299-309 (1986)

4 Sinaï Ya G. The limiting behavior of a one-dimensional random walk in random environment. Theory Probab Appl, 27: 256-268 (1982)

5 Comets F, Gantert N, Zeitouni O. Quenched, annealed and functional large deviations for one dimensional random walk in random environment. Probab Theory Related Fields, 118: 65-114 (2000)

6 Dembo A, Peres Y, Zeitouni O. Tail estimates for one-dimensional random walk in random environment. Comm Math Phys, 181: 667-683 (1996)

7 Gantert N, Zeitouni O. Quenched sub-exponential tail estimates for one-dimensional random walk in random environment. Comm Math Phys, 194: 177-190 (1998)

8 Greven A, den Hollander F. Large deviations for a random walk in random environment. Ann Probab, 22: 1381-1428 (1994)

9 Pisztora A, Povel T. Large deviation principle for random walk in a quenched random environment in the low speed regime. Ann Probab, 27: 1389-1413 (1999)

10 Pisztora A, Povel T, Zeitouni O. Precise large deviation estimates for a one-dimensional random walk in a random environment. Probab Theory Related Fields, 113: 191-219 (1999)

11 Sznitman A S. Topics in random walks in random environment. In: School and Conference on Probability Theory, ICTP Lect Notes, XVII. Trieste: Abdus Salam Int Cent Theoret Phys, 2004, 203-266

12 Zeitouni O. Random walks in random environment. XXXI Summer School in Probability (St. Flour, 2001), In: Lecture Notes in Mathematics, Vol. 1837. Berlin: Springer, 2004, 193-312

13 Zeitouni O. Random walks in random environments. J Phys A, 39(40): 433-464 (2006)

14 Bolthausen E, Goldsheid I. Recurrence and transience of random walks in random environments on a strip. Comm Math Phys, 214: 429-447 (2000)

15 Bolthausen E, Goldsheid I. Lingering random walks in random environment on a strip. Comm Math Phys, 278: 253-288 (2008) 
16 Brémont J. On some random walks on $\mathbb{Z}$ in random medium. Ann Probab, 30(2): 1-47 (2002)

17 Brémont J. Random walks in random medium on $\mathbb{Z}$ and Lyapunov spectrum. Ann Inst H Poincaré Probab Statist, 40: 309-336 (2004)

18 Goldsheid I. Linear and sub-linear growth and the CLT for hitting times of a random walk in random environment on a strip. Probab Theory Related Fields, 141(3-4): 471-511 (2008)

19 Le Page E. Théorèmes de renouvellement pour les produits de matrices aléatoires. Équations aux différences aléatoires. In: Séminaires de Probabilités Rennes. Rennes: Univ. Rennes I, 1983

20 Bougerol P, Lacroix J. Products of Random Matrices with Applications to Schrödinger Operators. Boston, MA: Birkhäuser, 1985

21 Hennion H, Hervé L. Limit Theorems for Markov Chains and Stochastic Properties of Dynamical Systems by Quasi-Compactness. Lecture Notes in Math, 1766. Berlin: Springer, 2001

22 Roitershtein A. A note on multitype branching processes with immigration in a random environment. Ann Probab, 35: 1573-1592 (2007)

23 Nagaev S V. Large deviations of sum of independent random variables. Ann Probab, 7: 749-789 (1979) 